T A B L E

Estimated Average number of extra Days, Average Amount of Extra Charges per infection, and Deaths CAUSed BY AND Contributed to BY Nosocomial INFections-United STATES

\begin{tabular}{|c|c|c|c|c|c|c|}
\hline \multirow[b]{2}{*}{ Type } & \multirow[b]{2}{*}{ Extra days } & \multirow[b]{2}{*}{ Extra charges' } & \multicolumn{2}{|c|}{$\begin{array}{c}\text { Deaths directly caused } \\
\text { by infections }\end{array}$} & \multicolumn{2}{|c|}{$\begin{array}{c}\text { Deaths to which } \\
\text { infections contributed }\end{array}$} \\
\hline & & & Total & $(\%)$ & Total & $(\%)$ \\
\hline Surgical wound infection & 7.3 & $\$ 3,152$ & 3,251 & $(0.6)$ & 9,726 & $(1.9)$ \\
\hline Lower respiratory tract infection & 5.9 & $\$ 5,683$ & 7,087 & $(3.1)$ & 22,983 & $(10.1)$ \\
\hline Bloodstream infection & 7.4 & $\$ 3,517$ & 4,496 & $(4.4)$ & 8,844 & $(8.6)$ \\
\hline Urinary tract infection & 1 & $\$ 680$ & 947 & $(0.1)$ & 6,503 & $(0.7)$ \\
\hline Other types & 4.8 & $\$ 1,617$ & 3,246 & $(0.8)$ & 10,036 & $(2.6)$ \\
\hline All types $\ddagger$ & 4 & $\$ 2,100$ & 19,027 & $(0.9)$ & 58,092 & $(2.7)$ \\
\hline
\end{tabular}

\section{Foodborne Outbreak of Escherichia coli O157:H7 Infections from Hamburgers -- Western United States, 1993}

During January 1993, 230 persons in the state of Washington were reported to have culture-confirmed infection with Escherichia coli $\mathrm{O} 157: \mathrm{H} 7$ resulting in bloody diarrhea and, in some cases, hemolytic uremic syndrome. Preliminary investigations linked cases to consumption of hamburgers from one fast-food restaurant chain. E coli $0157: \mathrm{H} 7$ has been isolated from epidemiologically implicated lots of ground beef, and an interstate recall was initiated by the restaurant on January 18. Additional possible cases and the source of the contaminated meat are still under investigation.

E coli $\mathrm{O} 157: \mathrm{H} 7$ was first linked to human illness in 1982, and its importance as a human pathogen appears to be increasing. A spectrum of illnesses have been associated with this organism, including mild diarrhea, severe bloody diarrhea (hemorrhagic colitis), hemolytic uremic syndrome often leading to acute renal failure, and death. Infection with this organism has been associated with consumption of contaminated beef and raw milk. Measures to prevent spread include thorough cooking of beef, pasteurization of milk, and careful handwashing with soap.

Diagnosis of $\mathrm{E}$ coli $0157: \mathrm{H} 7$ infection in the laboratory requires specific culture of stool specimens for the organism on modified MacConkey medium containing sorbitol.

This outbreak illustrates how surveillance with rapid reporting and prompt investigation of cases can lead to timely public health action. Physicians and laboratories are encouraged to report cases of $\mathrm{E}$ coli O157:H7 infection to their county and state health departments.
FROM: Centers for Disease Control and Prevention. Foodborne outbreak of Escherichia coli O157:H7 infections from hamburgers. M M WR. 1993;42:85-86.

\section{CDC Summarizes Progress on Control of Nosocomial Infections}

A recent report from the Centers for Disease Control and Prevention (CDC) in the M orbidity and $M$ ortality Weekly Report examined the knowledge about the effectiveness of nosocomial infection surveillance, prevention and control, and cost-benefits. The report says the annual cost for nosocomial infections is more than $\$ 4.5$ billion and involves more than 2 million patients each year. Adverse consequences of nosocomial infections and their associated costs vary by type of infection (Table).

Findings from the CDC's "Study on the Efficacy of Nosocomial Infection Control" (SENIC), conducted in the early 1970 s, was reviewed. SENIC found that hospitals reduced their nosocomial infection rates by approximately $32 \%$ if their programs included four components: 1) appropriate emphasis on surveillance activities and vigorous control efforts, 2) at least one full-time infection control practitioner per 250 beds, 3) a trained hospital epidemiologist, and 4) for surgical wound infections, feedback of wound infection rates to practicing surgeons.

SENIC established the effectiveness of infection control programs. However, other concerns regarding the cost-effectiveness and cost-benefit of such programs have emerged as the methods of reimbursement for U.S. hospitals have changed. Under the prospective payment system, virtually the entire cost of nosocomial infections represents an operating deficit. Thus, effective infection surveillance and control programs are the only way to reduce that cost. 